\title{
Intoxicação em cães e gatos: diagnóstico toxicológico empregando cromatografia em camada delgada e cromatografia líquida de alta pressão com detecção ultravioleta em amostras estomacais
}

\author{
Intoxication in dogs and cats: toxicological diagnosis using thin layer chromatography and high \\ pressure liquid chromatography with ultraviolet detection in stomach samples
}

\author{
Rachel Picada Bulcão ${ }^{\mathrm{I}, \mathrm{II}}$ Raquel TonelloII Sílvia Juliane Piva ${ }^{\text {II }}$ Gabriela Cristina Schmitt ${ }^{\mathrm{I}, \mathrm{II}}$ \\ Tatiana Emanuelli ${ }^{\mathrm{II}}$ Eliane Dallegrave ${ }^{\mathrm{IV}}$ Solange Cristina Garcia ${ }^{\mathrm{II}^{*}}$
}

RESUMO

Agrotóxicos e raticidas são responsáveis por inúmeras intoxicações humanas e animais. Dados preliminares sugerem que o uso ilegal desses compostos com a finalidade de intoxicação fatal em pequenos animais é uma prática comum na região central do Estado do Rio Grande do Sul. O Laboratório de Toxicologia (LATOX) recebe amostras de casos em que a principal suspeita é a intoxicação por agrotóxicos ou raticidas (lícitos e ilícitos). O presente estudo teve como objetivo apresentar um levantamento das intoxicações de pequenos animais, analisadas pelo LATOX no período de 2004 a 2008, sendo identificados os xenobióticos por meio de métodos analíticos otimizados pelo laboratório, incluindo screening por cromatografia em camada delgada (CCD) e possível confirmação por cromatografia líquida de alta eficiência (CLAE-UV). No período, foram analisadas 68 amostras oriundas de intoxicações em cães e gatos. As amostras biológicas analisadas foram o estômago e o conteúdo estomacal, das quais a CCD permitiu a identificação de carbamatos, warfarina e estricnina. Esta mostrou ser uma técnica qualitativa eficiente e adequada para esse propósito, além de ser relativamente rápida, de baixo custo e de sofrer pouca interferência de componentes da matriz. Também foi realizado um screening toxicológico por CLAE-UV para os carbamatos n-metilados: aldicarb, carbaril, carbofuran e propoxur. O resultado das análises indicou que o principal agente tóxico encontrado foi o aldicarb (chumbinho), responsável por 39,7\% das intoxicações (27 casos), seguido por estricnina (seis casos), warfarina (três casos) e monofluoracetato de sódio (um caso). Sendo assim, o "chumbinho" foi o principal agente envolvido em intoxicações de cães e gatos na região central do Estado no período avaliado, e os métodos analíticos CCD e CLAE-UV podem ser utilizados de forma eficiente na rotina laboratorial para identificação e confirmação dos xenobióticos mais envolvidos nessas intoxicações.

Palavras-chave: intoxicação, cães, gatos, CCD, CLAE, carbamatos.

\section{ABSTRACT}

The pesticides and rodenticides are responsible for several human and animal intoxications. Preliminary data suggest that the illegal use of these compounds to poison small animals is a common practice in the central region of the Rio Grande do Sul state. The Laboratory of Toxicology received samples, of cases in which the main suspected cause of death is poisoning by pesticides or exogenous rodenticides (licit and illicit). The aim of this study was show the survey of small animals poisoned and analyzed by LATOX during the period of 2004 to 2008 identifying the xenobiotics by optimized analytical methods, including screening by thin-layer chromatography (TLC) and a possible confirmation by high performance liquid chromatography - ultraviolet detection (HPLC-UV). In this period 68 samples were analyzed from small poisoned animals. The biological samples analyzed were stomach and stomach content and the TLC permitted carbamates, warfarin and stricnine identification. This proved to be an efficient and adequate technique for this purpose, relatively fast, inexpensive and with low matrix interference. The screening by HPLC for N-methyl carbamates was also realized: aldicarb, carbaryl, carbofuran and propoxur. The analysis showed that the main toxic agent found was aldicarb (chumbinho), responsible for 39.7\% of poisoning (27 cases), followed by stricnine (6 cases), warfarin (3 cases) and sodium

IPrograma de Pós-graduação em Ciências Farmacêuticas, Faculdade de Farmácia, Universidade Federal do Rio Grande do Sul (UFRGS), Porto Alegre, RS, Brasil.

IILaboratório de Toxicologia (LATOX), Departamento de Análises, Faculdade de Farmácia, UFRGS, Av. Ipiranga 2752, Bairro Santana, 90610-000, Porto Alegre, RS, Brasil. E-mail: garciapomblum@yahoo.com.br.*Autor para correspondência.

IIIDepartamento de Tecnologia dos Alimentos, Centro de Ciências Rurais (CCR), UFSM, Santa Maria, RS, Brasil.

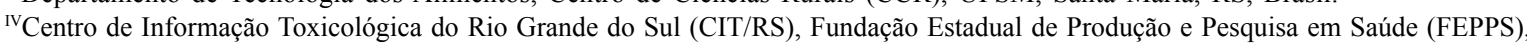
Porto Alegre, RS, Brasil. 
monofluoracetate (1 case). Thus, the 'chumbinho' was the main agent involved in dogs and cats poisoning in the central region of the state, during the analyzed period. The analytical methods TLC and HPLC can be used efficiently in laboratorial routine for identification and confirmation of xenobiotics involved in these poisonings.

Key words: poisoning, dogs, cats, TLC, HPLC, carbamate.

\section{INTRODUÇÃO}

Entre os anos de 1980 e 2006, foram registrados, no Centro de Informação Toxicológica do Rio Grande do Sul (CIT-RS), 10.400 casos de intoxicação em animais, sendo $72 \%$ das notificações em cães, e os principais agentes envolvidos foram pesticidas domésticos, produtos veterinários e também raticidas (DALLEGRAVE et al., 2007).

Os envenenamentos animais ocorrem, na maioria das vezes, por imprudência de proprietários que buscam alternativas para eliminar pragas, mesmo reconhecendo que algumas práticas são ilegais ou mesmo perigosas. Além disso, pessoas intoxicam propositadamente cães de guarda para facilitar o furto (ASSIS et al., 2009). Uma das principais causas de intoxicação pode ser considerada a desinformação da população quanto ao uso adequado dessas substâncias no ambiente doméstico, muitas vezes administradas ou utilizadas sem orientação ou acompanhamento de profissional qualificado, aumentando o risco de intoxicações (MEDEIROS et al., 2009).

A toxicologia moderna investiga uma ampla variedade de agentes que podem causar danos à saúde, sendo necessárias a avaliação precisa da exposição e a identificação do agente para se estimar o risco e subsidiar a terapêutica e as medidas profiláticas a serem tomadas (PESCH et al., 2004). Em estudos recentes, realizados em clínicas de pequenos animais e em unidades veterinárias, piretróides, organofosforados, carbamatos, cumarínicos e estricnina são apontados como os pesticidas mais envolvidos nessas ocorrências (XAVIER et al., 2002; ASSIS et al., 2009; MEDEIROS et al., 2009).

Muito já se conhece sobre a metodologia de extração e análise, em tecido e urina, de traços de vários compostos envolvidos em casos de intoxicação animal (PATIL \& SHINGARE, 1994; MARQUET et al., 1996); porém, não há muitos registros na literatura quando se trata de identificação dessas substâncias em conteúdo estomacal de animais domésticos (WANG et al., 2007; XAVIER et al., 2007). Sendo, portanto, essencial esse tipo de análise para identificação das intoxicações exógenas em animais.
Considerando que o Laboratório de Toxicologia tem recebido amostras de estômago e conteúdo gástrico de cães e gatos vítimas de intoxicações, o presente estudo objetivou avaliar as intoxicações exógenas fatais ocorridas em pequenos animais da região central do Estado, no período de 2004 e 2008, por meio da análise dessas amostras por cromatografia em camada delgada (CCD), para a detecção de carbamatos, warfarina e estricnina, e por cromatografia líquida de alta eficiência (CLAE), para diferentes carbamatos.

\section{MATERIAS E MÉTODOS}

As informações sobre as amostras avaliadas no presente estudo encontram-se registradas no livro de controle de amostras para análise e screening toxicológico no período de março de 2004 a março de 2008, no Laboratório de Toxicologia da Universidade Federal de Santa Maria (LATOX/UFSM).

Amostras: foram utilizadas 68 amostras de estômago obtidas durante a necropsia de cães e gatos recebidos pelo serviço de Patologia Veterinária da Universidade Federal de Santa Maria, que possuíam como hipótese diagnóstica a intoxicação exógena. A obtenção da amostra constou da abertura do estômago, seguida da lavagem estomacal, com água destilada (máximo 150mL), sendo retirado o conteúdo e raspada a parede do estômago, com posterior homogeneização do material. Todo o conteúdo gástrico foi coletado e conservado sob refrigeração $\left(4^{\circ} \mathrm{C}\right)$ por, no máximo, dois dias até a realização da análise.

Reagentes e padrões: os reagentes utilizados na extração e na CCD foram grau para análise (diclorometano, metanol, hexano, benzeno e hidróxido de amônio). Os solventes utilizados na CLAE, acetonitrila e metanol, foram o grau cromatográfico e a água purificada em sistema Milli-Q. Todos os padrões utilizados eram da marca Sigma-Aldrich ${ }^{\circledR}$.

Extração: após a lavagem e homogeneização do conteúdo gástrico, uma alíquota de $30 \mathrm{~mL}$ da amostra foi adicionada a $30 \mathrm{~mL}$ de uma solução de tampão tris ( $\mathrm{pH} 9,0)$ e extraída com $10 \mathrm{~mL}$ de diclorometano. A extração foi realizada em triplicata, e a fase orgânica foi filtrada com sulfato de sódio anidro, evaporada totalmente e ressuspendida com $250 \mu \mathrm{L}$ de metanol.

Cromatografia em camada delgada: após extração, as amostras foram cromatografadas contra os respectivos padrões em placa cromatográfica analítica de sílica gel $\mathrm{G}$, sendo utilizados benzeno: hexano: metanol: hidróxido de amônio (50:20:20:2) como eluente e solução de Draggendorf iodado como agente cromogênico. Foi realizada visualização com luz 
ultravioleta, já que alguns desses compostos absorvem nessa região, como, por exemplo, o aldicarb.

Cromatografia líquida de alta eficiência: foi utilizado um cromatógrafo líquido de alta eficiência com detecção ultravioleta (CLAE-UV) (Knauer, Berlim, Alemanha) equipado com uma bomba modelo K 1001, degasser online modelo K 5004, injetor manual com alça de amostragem de $20 \mu \mathrm{L}$, acoplado a um detector UV/VIS modelo K 2501. Foram empregadas condições cromatográficas que possibilitassem a identificação simultânea dos seguintes carbamatos $n$-metilados: aldicarb, carbaril e carbofuran (ë: $247 \mathrm{~nm}$ ). O propoxur, em razão de sua absorção em diferente comprimento de onda (ë: $270 \mathrm{~nm}$ ), foi identificado separadamente, mas sob as mesmas condições. A concentração utilizada para cada carbamato foi de $0,01 \mathrm{mg} \cdot \mathrm{ml}^{-1}$. As condições cromatográficas estão apresentadas na tabela 1 .

Cromatografia gasosa/detector por ionização de chama: análises para identificação de fluoracetato foram realizadas pelo Laboratório de Análises Toxicológicas Ltda de Porto Alegre, sendo utilizadas extração orgânica, derivatização metílica, extração etílica e análise por cromatografia gasosa/ detector por ionização de chama (CG/DIC).

\section{RESULTADOS E DISCUSSÃO}

Conforme os dados registrados na tabela 2, entre março de 2004 e março de 2008, foram realizadas 68 análises de estômago ou conteúdo estomacal de pequenos animais envolvidos em intoxicações fatais, das quais foram confirmadas $54,4 \%$, sendo $39,7 \%$ por carbamato, $4,4 \%$ por warfarina, $8,8 \%$ por estricnina e $1,4 \%$ por fluoroacetato de sódio. A incidência encontrada nesses casos de intoxicação revelou que os carbamatos foram os agentes tóxicos mais comumente relacionados. Além disso, dentre os carbamatos analisados por CLAE-UV, o "chumbinho" (aldicarb) foi o único identificado, sendo, portanto, o agente envolvido com mais frequência ( 27 casos) nessas ocorrências fatais em pequenos animais.
Dados epidemiológicos publicados por ASSIS et al. (2009), fundamentados em uma pesquisa realizada por meio de questionários aplicados em 44 estabelecimentos veterinários que atendem pequenos animais em Curitiba, durante um ano (2004/2005), revelaram que as intoxicações são caracterizadas por ter maior incidência em cães do que em gatos $(81 \% \mathrm{e}$ $18 \%$, respectivamente). Destes, destacam-se principalmente piretróides, organofosforados, carbamatos, cumarínicos e estricnina, sendo os carbamatos responsáveis por $56,3 \%$ dos óbitos registrados. Em razão da ampla disponibilidade de carbamatos no mercado consumidor, sendo legalmente comercializados como agrotóxicos, produtos veterinários ou mesmo como inseticidas domésticos, e da elevada toxicidade de alguns princípios ativos, como o aldicarb (DL50 oral para ratos $=1 \mathrm{mg} \mathrm{kg}^{-1}$ ), esses pesticidas são causadores de muitos casos de intoxicação por ingestão acidental ou mesmo intencional (ALMEIDA et al., 2005).

Estudos epidemiológicos também destacam que carbamatos são os principais agentes responsáveis pelo envenenamento de animais domésticos como cães e gatos. WANG et al. (2007) e XAVIER et al. (2007) publicaram estudos em que carbamatos foram indicados como sendo a principal causa de intoxicação aguda, acidental ou não. Segundo os autores, esse fato está relacionado a três fatores: 1) alta toxicidade de alguns compostos, principalmente o aldicarb $\left(\right.$ Temik $\left.^{\circledR}\right)$, um agente anticolinesterásico (carbamato) comercializado de forma clandestina e usado ilegalmente como raticida doméstico, 2) facilidade de aquisição de produtos registrados para uso agrícola contendo essas substâncias, e 3) fiscalização ainda ineficiente da comercialização dos agrotóxicos.

Atualmente, em grande parte do Brasil, o diagnóstico da intoxicação por carbamatos, warfarina, estricnina e fluoroacetato de sódio é baseado, principalmente, na história de exposição ou ingestão e nos sinais clínicos (ASSIS et al., 2009). Além disso, para a realização das análises toxicológicas, é necessário adequar as metodologias disponíveis às matrizes apresentadas.

Tabela 1 - Condições cromatográficas para análise de carbamatos n-metilados por CLAE-UV.

\begin{tabular}{|c|c|c|c|c|c|c|c|}
\hline Tipo de eluição & Fluxo & Fase móvel & Loop & Coluna & $\lambda_{1,2,3}$ & $\lambda_{4}$ & Tempo de análise \\
\hline \multirow{6}{*}{ Isocrática } & \multirow{6}{*}{$1 \mathrm{ml} \mathrm{min}^{-1}$} & B: $40 \%$ & \multirow{6}{*}{$20 \mu \mathrm{L}$} & \multirow{6}{*}{ C18 (ODS) } & \multirow{6}{*}{$247 \mathrm{~nm}$} & \multirow{6}{*}{$270 \mathrm{~nm}$} & \multirow{6}{*}{$1,2,3: 10 \mathrm{~min}$} \\
\hline & & Metanol & & & & & \\
\hline & & $\mathrm{C}: 25 \%$ & & & & & \\
\hline & & $\mathrm{ACN}: \mathrm{H} 2 \mathrm{O}$ & & & & & \\
\hline & & $(60: 40)$ & & & & & \\
\hline & & D: $35 \% \mathrm{H}_{2} \mathrm{O}$ & & & & & \\
\hline
\end{tabular}

Ciência Rural, v.40, n.5, mai, 2010. 
Tabela 2 - Incidência de intoxicações por carbamato, warfarina, estricnina e fluoroacetato de sódio em relação ao número total de suspeitas de intoxicação encaminhadas ao Laboratório de Toxicologia da Universidade Federal de Santa Maria no período de 2004 a 2008.

\begin{tabular}{|c|c|c|c|c|c|c|}
\hline Ano & $\begin{array}{l}\text { Suspeitas de } \\
\text { Intoxicação }\end{array}$ & Carbamato & Warfarina & Estricnina & $\begin{array}{l}\text { Fluoroacetato de } \\
\text { sódio }\end{array}$ & Porcentagem (\%) \\
\hline 2004 & 20 & 13 & 1 & - & - & 70 \\
\hline 2005 & 11 & 3 & 2 & 2 & - & 63,6 \\
\hline 2006 & 14 & 5 & - & 2 & - & 50 \\
\hline 2007 & 17 & 6 & - & 1 & - & 41,2 \\
\hline 2008 & 6 & - & - & 1 & 1 & 33,3 \\
\hline Total & 68 & 27 & 3 & 6 & 1 & 54,4 \\
\hline
\end{tabular}

Identificação cromatográfica dos xenobióticos

Dentre os métodos para análise química de agentes tóxicos, a cromatografia tem se mostrado como uma ferramenta valiosa para o isolamento e a identificação desses agentes em diversos tipos de amostras, incluindo as ambientais, embora existam poucos métodos simples para a identificação de agentes em amostras biológicas (CARDOSO et al., 2001).

A escolha do sistema eluente e do revelador para a realização da $\mathrm{CCD}$, para a detecção das substâncias mencionadas, foram fatores preponderantes. Foram realizadas pesquisas para identificar o melhor sistema solvente e o melhor revelador. A otimização do método permitiu a utilização de apenas um sistema eluente e de um revelador com sensibilidade adequada. Após esses testes, o sistema eluente escolhido foi benzeno:hexano:metanol:hidróxido de amônio (50:20:20:2). As placas foram posteriormente borrifadas com solução de Draggendorf iodado. O método foi otimizado a partir de MORAES et al. (1991). As condições cromatográficas para a detecção dos carbamatos, da warfarina e da estricnina foram idênticas. Os fatores de retenção (Rfs) encontrados foram 0,45 , 0,54, 0,50 e para os carbamatos aldicarb, carbaril e propoxur, respectivamente, 0,32 para a warfarina e 0,60 para a estricnina nessas condições. Além disso, no presente trabalho, todas as concentrações utilizadas foram capazes de produzir resultados positivos, mostrando manchas na mesma posição daquela produzida pelo padrão. A única diferença observada entre as diferentes concentrações testadas foi que a intensidade das manchas produzidas era diretamente proporcional à quantidade do xenobiótico presente na amostra. O carbofuran foi detectado apenas por CLAEUV. A visualização da placa cromatográfica na câmara de UV foi realizada antes da revelação com reagente de Draggendorf, auxiliando no screening para o aldicarb e confirmando sua visualização no fator de retenção de 0,45 .
As amostras com determinação somente para carbamatos foram analisadas por CLAE-UV, fazendo com que pequenas concentrações do xenobiótico presentes nas amostras fossem detectadas. Os tempos de retenção obtidos foram de 3,6min, 4,8min e 6,2min para aldicarb, carbaril e carbofuran, respectivamente, em $247 \mathrm{~nm}$ e 4,6 min para o propoxur, em 270nm.

A CCD tem sido empregada como método para a detecção de xenobióticos com finalidades forenses (AMENO et al., 2001; LACASSIE et al., 2001; CAZENAVE et al., 2005), podendo servir como um método qualitativo de triagem ou mesmo como ferramenta diagnóstica nos casos de intoxicação não fatais, quando a confirmação do diagnóstico facilita a escolha do melhor regime terapêutico.

Verificou-se a aplicabilidade da CCD como método de detecção de carbamatos, warfarina e estricnina, sendo empregados Sílica gel G como fase estacionária, benzeno:hexano:metanol:hidróxido de amônio (50:20:20:2) como fase móvel e solução de Draggendorf iodado como revelador (agente cromogênico). Além disso, a CLAE-UV tem sido descrita como método de escolha na determinação de pesticidas carbamatos, pois a maioria desses compostos não tem estabilidade térmica suficiente para a determinação por cromatografia gasosa (NUNES \& BARCELÓ, 1999).

O screening realizado com a utilização de CCD e CLAE-UV comprova que a associação dessas duas metodologias pode ser usada como ferramenta útil na elucidação de casos em que carbamatos $n$ metilados, mais especificamente o "chumbinho", tenha sido o responsável.

A confirmação do diagnóstico de intoxicação por análise laboratorial permite tornar estudos epidemiológicos e clínicos mais precisos. Tendo em vista a elevada utilização ilegal de agrotóxicos e raticidas sem os devidos cuidados no contexto estudado, o que favorece o aumento das intoxicações por pesticidas, a iniciativa da equipe do LATOX de 
otimizar métodos analíticos capazes de confirmar as suspeitas clínicas de envenenamentos fatais em pequenos animais é de fundamental importância no contexto toxicológico atual em veterinária.

\section{CONCLUSÃO}

A análise das amostras de conteúdo estomacal de pequenos animais vítimas de intoxicações exógenas fatais ocorridas na região central do Rio Grande do Sul, no período de 2004 e 2008, permitiu, para a maioria dos casos avaliados, firmar o diagnóstico de que carbamatos, principalmente o aldicarb, a warfarina e a estricnina foram os agentes mais envolvidos nessas ocorrências. Além disso, foi verificado que houve correlação entre os achados de necropsia sugestivos de intoxicação e a presença dos referidos agentes tóxicos nas amostras analisadas cromatograficamente (CCD e CLAE-UV) e, portanto, que as metodologias analíticas utilizadas para a identificação desses xenobióticos em conteúdo estomacal mostraram-se efetivas e úteis para auxílio diagnóstico.

Sendo assim, o presente estudo apresenta alternativas de adequar a metodologia analítica às necessidades dos profissionais envolvidos no diagnóstico e na terapêutica dessas injúrias, permitindo, assim, contribuir com estudos futuros, bem como fundamentar a necessidade de se estabelecer ações preventivas mais eficazes.

\section{AGRADECIMENTOS}

Ao Conselho Nacional de Desenvolvimento Científico e Tecnológico $(\mathrm{CNPq})$, pela bolsa de pesquisador concedida para Dra. Solange. C. Garcia.

\section{REFERÊNCIAS}

ALMEIDA, C.G. et al. Intoxicação em cães e gatos: estudo retrospectivo dos casos atendidos em um centro veterinário privado durante o período de 1996 a 2004. Revista Brasileira de Toxicologia, v.18, p.286, 2005.

AMENO, K. et al. Blood carbofuran concentrations in suicidal ingestion. Forensic Science International, v.116, p.59-61, 2001. Disponível em: <http://linkinghub.elsevier.com/retrieve/ pii/S0379073800003364>. Acesso em: 20 mai. 2008. doi:10.1016/S0379-0738(00)00336-4

ASSIS, H.C.S. et al. Perfil das intoxicações apresentadas por cães e gatos em Curitiba, Paraná. Revista do Conselho Federal de Medicina Veterinária, v.15, n.47, p.22-28, 2009.

CARDOSO, M.F.E.C. et al. Laboratório. In: ANDRADE FILHO, A.; CAMPOLINA, D. (Eds.). Toxicologia na prática clínica. Belo Horizonte: Folium, 2001. p.323-328.

CAZENAVE, S.O.S. et al. Aldicarb: uma possibilidade de análise com finalidade forense. Revista Brasileira de Toxicologia, v. 18, p.105-111, 2005.
DALLEGRAVE, E. et al. Toxicologia veterinária: do desenvolvimento sustentável à melhoria da qualidade de vida. In: RIO GRANDE DO SUL. Secretaria da Saúde. Fundação Estadual de Produção e Pesquisa em Saúde. Centro de Informação Toxicológica. Toxicovigilância - Toxicologia clínica: dados e indicadores selecionados, Rio Grande do Sul: 2006. Porto Alegre: CIT/RS, 2007. p.11-17.

LACASSIE, E. et al. Sensitive and specific multiresidue methods for the determination of pesticides of various classes in clinical and forensic toxicology. Forensic Science International, v.121, p.116-125, 2001. Disponível em: <http://linkinghub.elsevier.com/ retrieve/pii/S0379073801004613>. Acesso em: nov. 2007. doi:10.1016/S0379-0738(01)00461-3

MARQUET, P. et al. Analytical findings in a suicide involving sodium azide. Journal of Analytical Toxicology, v.20, n.2, p.93-100, 1996

MEDEIROS, R.J. et al. Casos de intoxicações exógenas em cães e gatos atendidos na Faculdade de Veterinária da Universidade Federal Fluminense durante o período de 2002 a 2008. Ciência Rural, Santa Maria, v.39, n.7, p.2105-2110 2009. Disponível em: <http:/ /www.scielo.br/scielo.php? script $=$ sci_arttext\&pid $=\mathrm{S} 010$ $384782009000700023 \& \operatorname{lng}=\mathrm{en} \& \mathrm{nrm}=\mathrm{iso}>$. Acesso em: 30 nov. 2009. doi: 10.1590/S0103-84782009005000151.

MORAES, E.C.F. et al. Manual de toxicologia analítica. São Paulo: Roca, 1991. 229p.

NUNES, G.S.; BARCELÓ, D. Analysis of carbamate insecticides in foodstuffs using chromatography and immunoassay techniques. Trends in Analytical Chemistry, v.18, n.2, p.99107, 1999. Disponível em: <http://dx.doi.org/10.1016/S01659936(98)00076-4>. Acesso em: 29 nov. 2007. doi:10.1016/ S0165-9936(98)00076-4.

PATIL, V.B.; SHINGARE, M.S. Thin-layer chromatographic spray reagent for the screening of biological materials for the presence of carbaryl. Analyst, v.119, p.415-416, 1994.

PESCH, B. et al. Challenges to environmental toxicology and epidemiology: where do we stand and which way do we go? Toxicology Letters, v.252, p.255-265, 2004.

WANG, Y. et al. Pesticide poisoning in domestic animals and livestock in Austria: a 6 years retrospective study. Forensic Science International, v.169, n.2-3, p.157-160, 2007.

XAVIER, F.G. et al. Toxicologia do praguicida aldicarb («chumbinho»): aspectos gerais, clínicos e terapêuticos em cães e gatos. Ciência Rural, v.37, n.4, p.1206-1211, 2007. Disponível em: <http:// www.scielo.br/scielo.php?script $=$ sci_arttext\&pid $=\mathrm{S} 0103$ $84782007000400051>$. Acesso em: 15 jul. 2008. doi: 10.1590/ S0103-84782007000400051.

XAVIER, F.G. et al. Cromatografia em camada delgada para o diagnóstico da intoxicação por aldicarb («chumbinho») em cães e gatos. Arquivo Brasileiro de Medicina Veterinária e Zootecnia, Belo Horizonte, v.59, n.5, 2007. Disponível em: $<$ http://www.scielo.br/ scielo.php?script $=$ sci_arttext\&pid $=\mathrm{S} 01$ $0209352007000500020 \& \operatorname{lng}=\mathrm{en} \& \mathrm{nrm}=\mathrm{iso}>$. Acesso em: 20 ago. 2009. doi: 10.1590/S0102-09352007000500020.

Ciência Rural, v.40, n.5, mai, 2010. 